\title{
Ising chain with topological degeneracy induced by dissipation
}

\author{
K. L. Zhang and Z. Song* \\ School of Physics, Nankai University, Tianjin 300071, China
}

(Dated: June 24, 2020)

\begin{abstract}
The ground-state degeneracy of the quantum spin system is a characteristic of nontrivial topology, when it is gapped and robust against disordered perturbation. The corresponding quantum phase transition (QPT) is usually driven by a real parameter. We study a non-Hermitian Ising chain with two transverse fields, one real and another imaginary, based on the exact solution and numerical simulation. We show that topological degeneracy still exists and can be obtained by an imaginary transverse field from a topologically trivial phase of a Hermitian system. The topological degeneracy is robust against the random imaginary field and therefore expected to be immune to disordered dissipation from the spontaneous decay in experiment. The underlying mechanism is the nonlocal symmetry, which emerges only in thermodynamic limit and relates two categories of QPTs in the quantum spin system, rooted from topological order and symmetry breaking, respectively.
\end{abstract}

\section{INTRODUCTION}

Driving a quantum phase transition (QPT) is of interest to both condensed matter physics and quantum information science. A varying parameter across the critical point induces a symmetry spontaneous breaking for traditional $\mathrm{QPT}^{1}$ and nonlocal topological order for topological $\mathrm{QPT}^{2,3}$. In the recent works ${ }^{4,5}$, it turns out that the local order parameter and the topological order parameter can coexist to characterize the QPTs. The underlying mechanism of this fact is the duality of the Kitaev model, which has been introduced to describe one-dimensional spinless fermions with superconducting $p$-wave pairing ${ }^{6}$. On the one hand, It is the fermionized version of the familiar one-dimensional (1D) transverse-field Ising $\operatorname{model}^{7}$, which is one of the simplest solvable models exhibiting quantum criticality and demonstrating a QPT with spontaneous symmetry breaking $^{1}$. On the other hand, as the gene of a Kitaev model, its Majorana lattice is the Su-SchriefferHeeger (SSH) model ${ }^{8}$, which has served as a paradigmatic example of the $1 \mathrm{D}$ system supporting topological character $^{9}$. It manifests the typical feature of topological order since the number of zero energy and edge states are immune to local perturbations ${ }^{10}$. The topological superconducting has been demonstrated by unpaired Majorana modes exponentially localized at the ends of open Kitaev chains, which are robust against disordered perturbation. A system with topological phase can be a promising platform for quantum computation and information processing due to the intrinsic stability of the topological feature ${ }^{11-13}$.

So far, most of the investigations on the QPT are driven by varying a real parameter at absolute zero temperature. However, in practice, a genuine quantum system, such as cold atoms, is intrinsically non-Hermitian because of spontaneous decay ${ }^{14-19}$. On the other hand, a non-Hermitian Hamiltonian is no longer a forbidden regime in quantum mechanics since the discovery that a certain class of non-Hermitian Hamiltonians could exhibit entirely real spectra ${ }^{20-23}$. It also turns out that certain types of non-Hermitian terms may maintain the topological feature of the original Hermitian system ${ }^{24}$. A natural question is whether a topologically trivial phase of a Hermitian system can be shifted to a nontrivial phase with stable ground states by adding a nonHermitian term.

In this paper, we investigate a one-dimensional quantum Ising model with a complex transverse field. The aim of this paper is to study the consequence of an imaginary transverse field on the topology of the ground state. Intuitively, the imaginary transverse field may break the Hermiticity of the Hamiltonian. However, it is shown that the non-Hermitian Ising model can be mapped to a Hermitian Ising model in the context of a biorthogonal inner product. This allows us to employ the same way for the Hermitian system, i.e., the robust degeneracy of ground states, to identify the nature of quantum phases. We show that the topological degeneracy still exists in the presence of a complex transverse field. We obtain the non-Hermitian version of the mapping operator to connect two degenerate ground states in the topologically nontrivial region. In addition, numerical simulation for a finite-size system indicates the existence of topological degeneracy since the degeneracy cannot be lifted by disordered perturbation on the imaginary transverse field. Importantly, the Hamiltonian has a full real spectrum in two different quantum phases. This property promises the stable ground states, and allows the theoretical and experimental investigations for the dynamical control and testing of the QPT.

This paper is organized as follows. In Sec. II, we present the model and its Hermitian counterpart. In Sec. III, we analyze the topological degeneracy in the Hermitian version. In Sec. IV, we propose the concept of nonlocal symmetry and numerically study the robustness against the disordered imaginary field. In Sec. V, we summarize the results and explore their implications. 


\section{HAMILTONIAN AND HERMITIAN COUNTERPART}

We start our investigation by considering a nonHermitian Ising chain with a complex transverse field

$$
H=-\sum_{j=1}^{N-1} \sigma_{j}^{x} \sigma_{j+1}^{x}+\sum_{j=1}^{N} g_{j}\left(\sigma_{j}^{z}+i \gamma_{j} \sigma_{j}^{y}\right),
$$

where $\sigma_{j}^{\lambda}(\lambda=x, y, z)$ are the Pauli operators on site $j$, and $g_{j}$ and $\gamma_{j}(i=\sqrt{-1})$ are real numbers. The nonHermitian Hamiltonian $H$ has pseudo-Hermiticity: either it has a real spectrum or else its complex eigenvalues always come in complex conjugate pairs ${ }^{20}$. As far as we know, although non-Hermitian systems indeed have some peculiar features and some of them have been proved to be equivalent to Hermitian systems under some particular conditions ${ }^{25-30}$, the complex field is always seen as unphysical. Recently some works, including theoretical and experimental research on Lee Yang zeros, which are the points on the complex plane of physical parameters, have been proposed ${ }^{31-37}$. They relate a complex field to the real world to some extent. On the other hand, it was proposed that ${ }^{19}$ an imaginary transverse field can be implemented by optically pumping a qubit state into the auxiliary state with a scheme similar to heralded entanglement protocols.

In order to explore the property of the non-Hermitian model, we introduce a transformation

$$
\begin{aligned}
\tau_{j}^{x} & =\sigma_{j}^{x}, \\
\tau_{j}^{y} & =\eta_{j}^{+} \sigma_{j}^{y}-i \eta_{j}^{-} \sigma_{j}^{z}, \\
\tau_{j}^{z} & =\eta_{j}^{+} \sigma_{j}^{z}+i \eta_{j}^{-} \sigma_{j}^{y},
\end{aligned}
$$

where the factors are $\eta_{j}^{+}=1 / \sqrt{1-\gamma_{j}^{2}}$ and $\eta_{j}^{-}=$ $\gamma_{j} / \sqrt{1-\gamma_{j}^{2}}$. The new spin operators still satisfy the Lie algebra commutation relations

$$
\left[\tau_{j}^{\mu}, \tau_{j}^{\nu}\right]=\sum_{\lambda=x, y, z} 2 i \epsilon^{\mu \nu \lambda} \tau_{j}^{\lambda},
$$

although $\tau_{j}^{y}$ and $\tau_{j}^{z}$ are not Hermitian. Applying the transformation on the Hamiltonian $H$, we have

$$
\mathcal{H}=-\sum_{j=1}^{N-1} \tau_{j}^{x} \tau_{j+1}^{x}+\sum_{j=1}^{N} g_{j} \sqrt{1-\gamma_{j}^{2}} \tau_{j}^{z} .
$$

Hamiltonian $\mathcal{H}$ represents an Ising model with a real transverse filed if $\left|\gamma_{j}\right|<1$, and it has full real spectrum although the spin operators $\tau_{j}^{y}$ and $\tau_{j}^{z}$ are not Hermitian. Within this region, $\mathcal{H}$ shares the same properties of $H$ with $\gamma_{j}=0$, in the context of the biorthogonal inner product. The connection between the eigenstates of $H$ and $\mathcal{H}$ can be obtained from the following relations

$$
| \pm\rangle_{j}=\frac{1}{\sqrt{2}}\left(\xi^{ \pm}|\uparrow\rangle_{j}-\frac{\gamma}{\xi^{ \pm}}|\downarrow\rangle_{j}\right),
$$

where $\xi^{ \pm}=\sqrt{1 \pm \sqrt{1-\gamma^{2}}}$, and the corresponding local spin vectors are defined as

$$
\begin{aligned}
\tau_{j}^{z}| \pm\rangle_{j} & = \pm| \pm\rangle_{j}, \\
\sigma_{j}^{z}|\uparrow\rangle_{j} & =|\uparrow\rangle_{j}, \sigma_{j}^{z}|\downarrow\rangle_{j}=-|\downarrow\rangle_{j} .
\end{aligned}
$$

Accordingly, the biorthonomal complete set of eigenstates can be established by the eigenstates of $\mathcal{H}^{\dagger}$.

The aim of this paper is to investigate the effect of $\left\{\gamma_{j}\right\}$ on the topological feature of quantum phases. Now we know that when an imaginary transverse field $\left\{\gamma_{j}\right\}$ is applied, the non-Hermitian Hamiltonian has a Hermitian counterpart, which is the original Ising model with a real transverse field shifted by a $\gamma_{j}$-related amount, i.e., $g_{i} \rightarrow g_{i} \sqrt{1-\gamma_{j}^{2}}$. In this sense, an imaginary field can lead to QPT. According to quantum theory of QPT in a Hermitian system, a second-order QPT is characterized by the divergence of the ground-state energy density. For the Hamiltonian $H$ in Eq. (1) with $g_{i}=g$ and $\gamma_{i}=\gamma=0$, we have

$$
\lim _{g \rightarrow 1} \frac{\partial^{2} \varepsilon_{\mathrm{g}}}{\partial g^{2}}=\infty
$$

where $\varepsilon_{\mathrm{g}}$ is the density of the ground-state energy. Meanwhile for the Hamiltonian $\mathcal{H}$ in Eq. (4) with $g_{i}=g$ and $\gamma_{i}=\gamma$, we have

$$
\frac{\partial \varepsilon_{\mathrm{g}}}{\partial \gamma}=-\frac{\gamma}{\sqrt{1-\gamma^{2}}} \frac{\partial \varepsilon_{\mathrm{g}}}{\partial \sqrt{1-\gamma^{2}}},
$$

which results in

$$
\lim _{\gamma \rightarrow \gamma_{c}} \frac{\partial^{2} \varepsilon_{\mathrm{g}}}{\partial \gamma^{2}}=\infty
$$

due to the replacement $g \rightarrow g \sqrt{1-\gamma^{2}}$. It indicates that $\gamma$ drives a second-order QPT at $\gamma_{c}= \pm \sqrt{1-g^{-2}}$. In the next two sections, we will analyze this issue from the topological aspect.

\section{GROUND-STATE DEGENERACY AND EDGE MODES}

We first revisit the connection between ground-state degeneracy and Majorana edge modes in the Hermitian transverse field Ising chain in this section, and then we extend the conclusion to the non-Hermitian version in the next section. Consider the Hamiltonian $H$ in Eq. (1) with $g_{i}=g$ and $\gamma_{i}=\gamma=0$,

$$
H_{\text {spin }}=-\sum_{j=1}^{N-1} \sigma_{j}^{x} \sigma_{j+1}^{x}+g \sum_{j=1}^{N} \sigma_{j}^{z},
$$

which is a standard Ising model with an open boundary condition. The $2^{N}$-dimensional complete set of basis can be constructed by applying operators $\sigma_{j}^{+}=\left(\sigma_{j}^{x}+i \sigma_{j}^{y}\right) / 2$ 
on a saturated ferromagnetic state $\prod_{j=1}^{N}|\downarrow\rangle_{j}$. The whole Hilbert space can be decomposed into two invariant subspaces, with even and odd numbers of spin flips from the above ferromagnetic state. Then all the eigenstates of $H_{\text {spin }}$ can be classified into two groups, denoted as $\left\{\left|\psi_{j}^{+}\right\rangle\right\}$and $\left\{\left|\psi_{j}^{-}\right\rangle\right\}$, respectively. In the following, we show the connection between two such groups of eigenstates, and the implication for topological degeneracy.

As a standard procedure, one can perform the JordanWigner transformation ${ }^{38}$

$$
\begin{aligned}
\sigma_{j}^{x} & =\prod_{l<j}\left(1-2 c_{l}^{\dagger} c_{l}\right)\left(c_{j}+c_{j}^{\dagger}\right), \\
\sigma_{j}^{y} & =i \prod_{l<j}\left(1-2 c_{l}^{\dagger} c_{l}\right)\left(c_{j}-c_{j}^{\dagger}\right), \\
\sigma_{j}^{z} & =2 c_{j}^{\dagger} c_{j}-1,
\end{aligned}
$$

to replace the Pauli operators by the fermion operators $c_{j}$. In this paper, we focus on the system with an open boundary condition, in which there are no differences between even and odd numbers of fermions. The Hamiltonian is transformed to a Kitaev model

$$
\begin{aligned}
H_{\text {Kitaev }}= & -\sum_{j=1}^{N-1}\left(c_{j}^{\dagger} c_{j+1}+c_{j}^{\dagger} c_{j+1}^{\dagger}\right)+\text { H.c. } \\
& +g \sum_{j=1}^{N}\left(2 c_{j}^{\dagger} c_{j}-1\right) .
\end{aligned}
$$

We note that the parity of particles number is conservative, i.e., $\left[(-1)^{\sum_{j=1}^{N} c_{j}^{\dagger} c_{j}}, H_{\text {Kitaev }}\right]=0$, corresponding to the classification of the eigenstates, $\left\{\left|\psi_{j}^{+}\right\rangle\right\}$and $\left\{\left|\psi_{j}^{-}\right\rangle\right\}$.

To get the solution of the model, one can introduce the Majorana fermion operators $a_{j}=c_{j}^{\dagger}+c_{j}$ and $b_{j}=$ $-i\left(c_{j}^{\dagger}-c_{j}\right)$, and then obtain the Majorana representation of the Hamiltonian, the core matrix of which is that of a $2 N$-site $\mathrm{SSH}$ chain in a single-particle invariant subspace. Based on the exact diagonalization results of the SSH chain, the Hamiltonian $H_{\text {Kitaev }}$ can be written as the diagonal form

$$
H_{\text {Kitaev }}=\sum_{n=1}^{N} \varepsilon_{n}\left(d_{n}^{\dagger} d_{n}-\frac{1}{2}\right) .
$$

Here $d_{n}$ is the fermonic operator, satisfying $\left\{d_{n}, d_{n^{\prime}}\right\}=0$, and $\left\{d_{n}, d_{n^{\prime}}^{\dagger}\right\}=\delta_{n, n^{\prime}}$. The spectrum $\varepsilon_{n}$ and the explicit expression of $d_{n}$ can be obtained by the diagonalization of the core matrix:

$$
\begin{aligned}
M_{\mathrm{SSH}}= & -\frac{1}{2} \sum_{j=1}^{N-1}|2 j\rangle\langle 2 j+1| \\
& +\frac{1}{2} g \sum_{j=1}^{N}|2 j-1\rangle\langle 2 j|+\text { H.c. }
\end{aligned}
$$

On the other hand, no matter what the explicit solution is, we always have the relations

$$
\left[d_{n}, H_{\text {Kitaev }}\right]=\varepsilon_{n} d_{n},\left[d_{n}^{\dagger}, H_{\text {Kitaev }}\right]=-\varepsilon_{n} d_{n}^{\dagger},
$$

which result in the mapping between the eigenstates of $H_{\text {Kitaev }}$. For an arbitrary eigenstate $|\psi\rangle$ of $H_{\text {Kitaev }}$ with the eigenenergy $E$, i.e.,

$$
H_{\text {Kitaev }}|\psi\rangle=E|\psi\rangle
$$

state $d_{n}|\psi\rangle\left(d_{n}^{\dagger}|\psi\rangle\right)$ is also an eigenstate of $H_{\text {Kitaev }}$ with the eigenenergy $E-\varepsilon_{n}\left(E+\varepsilon_{n}\right)$, i.e.,

$$
H_{\text {Kitaev }}\left(d_{n}|\psi\rangle\right)=\left(E-\varepsilon_{n}\right)\left(d_{n}|\psi\rangle\right),
$$

and

$$
H_{\text {Kitaev }}\left(d_{n}^{\dagger}|\psi\rangle\right)=\left(E+\varepsilon_{n}\right)\left(d_{n}^{\dagger}|\psi\rangle\right)
$$

if $d_{n}|\psi\rangle \neq 0\left(d_{n}^{\dagger}|\psi\rangle \neq 0\right)$.

In this paper, we are interested in the topological degeneracy in the topologically non-trivial phase, which arises from two zero-eigenenergy edge states of the matrix in Eq. (14) for $|g|<1$ in the large $N$ limit. Actually, in small $N$, the energies of the edge states are not exactly zero, but are exponentially small in $N^{10}$. However, here we are interested in the second-order QPT in the large $N$ limit, thus the terms that are exponentially small in $N$ are ignored in the following discussion. It turns out that within the topological region, the edge modes appear with $\varepsilon_{N}=0$ and the edge operator $d_{N}$ can be expressed as

$$
\begin{aligned}
d_{N}= & \frac{1}{2} \sqrt{1-g^{2}} \sum_{j=1}^{N}\left[\left(g^{j-1}+g^{N-j}\right) c_{j}^{\dagger}\right. \\
& \left.+\left(g^{j-1}-g^{N-j}\right) c_{j}\right]
\end{aligned}
$$

i.e., $d_{N}$ is a linear combination of particle and hole operators of spinless fermions $c_{j}$ on the edge. Furthermore, applying the inverse Jordan-Wigner transformation, we can express $d_{N}$ as the combination of spin operators:

$$
\begin{aligned}
D_{N}= & \frac{1}{2} \sqrt{1-g^{2}} \sum_{j=1}^{N} \prod_{l<j}\left(-\sigma_{l}^{z}\right)\left[\left(g^{j-1}+g^{N-j}\right) \sigma_{j}^{+}\right. \\
& \left.+\left(g^{j-1}-g^{N-j}\right) \sigma_{j}^{-}\right] .
\end{aligned}
$$

In fact, $d_{N}$ and $D_{N}$ are identical, but only in different representations. Obviously, from $\left[d_{N}, H_{\text {Kitaev }}\right]=0$, we have

$$
\left[D_{N}, H_{\text {spin }}\right]=\left[D_{N}^{\dagger}, H_{\text {spin }}\right]=0,
$$

which leads to the degeneracy of the eigenstates. The $D_{N}$ operator can also be obtained by using the iterative method ${ }^{39,40}$. The properties of the fermion operator $D_{N}$ are similar to that of the "strong edge zero mode" $\Psi$, which however, is a Majorana fermion operator ${ }^{39-41}$. Moreover, the operator $D_{N}$ can be obtained by the left 
and right "strong edge zero modes" $\Psi_{\mathrm{L} / \mathrm{R}}$, i.e., $D_{N}=$ $\left(\Psi_{\mathrm{L}}+i \Psi_{\mathrm{R}}\right) / 2$. We would like to point out that the commutation relation in Eq. (21) can be regarded as the symmetry of the system. Importantly, such a symmetry is conditional, requiring $|g|<1$ in the large $N$ limit. This accords with the symmetry breaking mechanism for $\mathrm{QPT}^{1}$. On the other hand, the QPT also has topological characteristics since the edge mode is robust against disorder perturbation. Here we do not review this content in the Hermitian regime, but we investigate it directly in the non-Hermitian regime in the next section.

\section{NONLOCAL SYMMETRY AND ROBUSTNESS OF DEGENERACY}

Starting from the non-Hermitian Hamiltonian $H$ in Eq. (1), it is tough to find out the mapping operator along the same route in the last section ${ }^{42,43}$. However, we note that the commutation relation in Eq. (3) is only based on the Lie algebra commutation relation of spin operators $\left\{\sigma_{j}^{\alpha}\right\}$ no matter whether they are Hermitian or non-Hermitian. Then one can construct the mapping operator directly by replacing $\left\{\sigma_{j}^{\alpha}\right\}$ with $\left\{\tau_{j}^{\alpha}\right\}$. In parallel, we have the mapping operator of the non-Hermitian version

$$
\begin{aligned}
\mathcal{D}_{N}= & \frac{1}{2} \sqrt{1-\mathfrak{g}^{2}} \sum_{j=1}^{N} \prod_{l<j}\left(-\tau_{l}^{z}\right)\left[\left(\mathfrak{g}^{j-1}+\mathfrak{g}^{N-j}\right) \tau_{j}^{+}\right. \\
& \left.+\left(\mathfrak{g}^{j-1}-\mathfrak{g}^{N-j}\right) \tau_{j}^{-}\right],
\end{aligned}
$$

and its canonical conjugation

$$
\begin{aligned}
\overline{\mathcal{D}}_{N}= & \frac{1}{2} \sqrt{1-\mathfrak{g}^{2}} \sum_{j=1}^{N} \prod_{l<j}\left(-\tau_{l}^{z}\right)\left[\left(\mathfrak{g}^{j-1}+\mathfrak{g}^{N-j}\right) \tau_{j}^{-}\right. \\
& \left.+\left(\mathfrak{g}^{j-1}-\mathfrak{g}^{N-j}\right) \tau_{j}^{+}\right],
\end{aligned}
$$

in the large $N$ limit, where the equivalent real field $\mathfrak{g}=$ $g \sqrt{1-\gamma^{2}}$ and $\mathcal{D}_{N}\left(\overline{\mathcal{D}}_{N}\right)$ is only applicable within the region $\mathfrak{g}<1$, or $1>\gamma>\gamma_{c}$ with critical $\gamma_{c}=\sqrt{1-g^{-2}}$. When $\gamma \neq 0$, we note that $\mathcal{D}_{N}^{\dagger} \neq \overline{\mathcal{D}}_{N}$ due to the nonHermitian nature of $\tau_{j}^{ \pm}$. At $\gamma=1$, the system is at the exceptional point, which is beyond our investigation. Obviously, we still have

$$
\left[\mathcal{D}_{N}, \mathcal{H}\right]=\left[\overline{\mathcal{D}}_{N}, \mathcal{H}\right]=0
$$

and the canonical commutation relations

$$
\left\{\mathcal{D}_{N}, \overline{\mathcal{D}}_{N}\right\}=1,\left(\mathcal{D}_{N}\right)^{2}=\left(\overline{\mathcal{D}}_{N}\right)^{2}=0
$$

which guarantee the existence of degeneracy of the eigenstates. Applying the operators on the lowest energy eigenstates $\left|\psi_{\mathrm{g}}^{+}\right\rangle$and $\left|\psi_{\mathrm{g}}^{-}\right\rangle$in two invariant subspaces, we have

$$
\begin{aligned}
& \mathcal{D}_{N}\left|\psi_{\mathrm{g}}^{+}\right\rangle=\left|\psi_{\mathrm{g}}^{-}\right\rangle, \overline{\mathcal{D}}_{N}\left|\psi_{\mathrm{g}}^{-}\right\rangle=\left|\psi_{\mathrm{g}}^{+}\right\rangle \\
& \overline{\mathcal{D}}_{N}\left|\psi_{\mathrm{g}}^{+}\right\rangle=\mathcal{D}_{N}\left|\psi_{\mathrm{g}}^{-}\right\rangle=0
\end{aligned}
$$

and then $\left|\psi_{\mathrm{g}}^{+}\right\rangle$and $\left|\psi_{\mathrm{g}}^{-}\right\rangle$are degenerate ground states. This indicates that the existence of such a degeneracy depends on the value of $\gamma$ through $\mathfrak{g}$. In Fig. 1 we demonstrate this feature by the plots of the lower-energy levels of $\mathcal{H}$ on finite size, as a function of $\gamma$. We can see that as $\gamma$ increases, the energy gap closes at the pseudocritical point, and states $\left|\psi_{\mathrm{g}}^{+}\right\rangle$and $\left|\psi_{\mathrm{g}}^{-}\right\rangle$turn to degenerate. Meanwhile, many pairs of excited states also become degenerate near the pseudocritical point, as expected in Eq. (24).

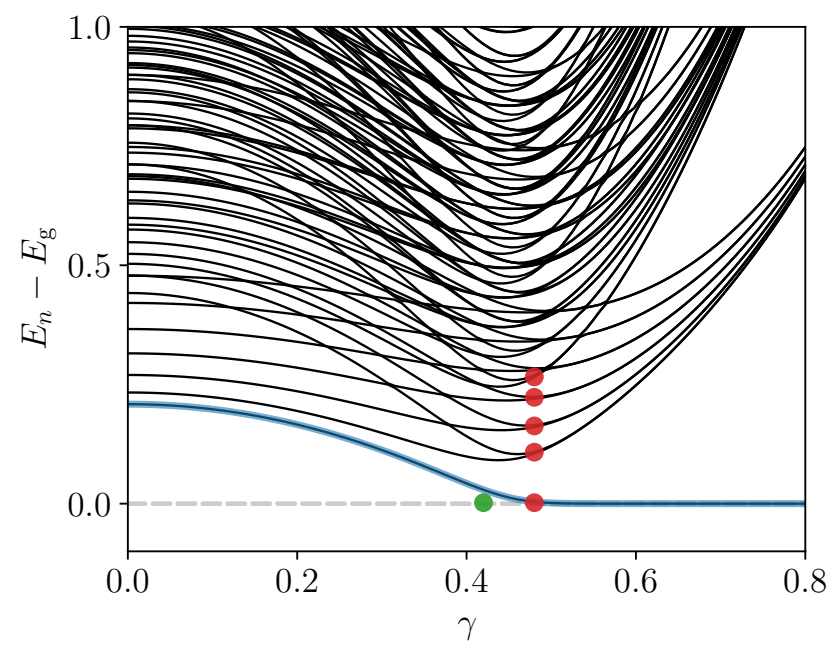

FIG. 1. The lower-energy levels (subtracted by the groundstate energy) of $\mathcal{H}$ as a function of $\gamma$, obtained by exact diagonalization numerically. Parameters of the system are $N=100$ and $g=1.1$. The energy gap between two ground states of two invariant subspaces, $\left|\psi_{\mathrm{g}}^{+}\right\rangle$and $\left|\psi_{\mathrm{g}}^{-}\right\rangle$, vanishes at the pseudocritical point $\gamma=0.48$, marked by a red dot, which is closed to the critical point in the thermodynamic limit, $\gamma_{c}=\sqrt{1-g^{-2}} \approx 0.42$, marked by a green dot. It indicates that the imaginary transverse field can drive the second-order QPT. In addition, we can see that there are many pairs of excited states, which also turn to degenerate near the pseudocritical point marked by the red dots. It accords with the prediction obtained by the existence of nonlocal symmetry in Eq. (24).

Now we turn to investigate the performance of the ground states $\left|\psi_{\mathrm{g}}^{ \pm}\right\rangle$as the imaginary field is disordered. Consider a model with random parameters

$$
\mathcal{H}_{\text {Ran }}=-\sum_{j=1}^{N-1} \sigma_{j}^{x} \sigma_{j+1}^{x}+g \sum_{j=1}^{N}\left(\sigma_{j}^{z}+i \gamma_{j} \sigma_{j}^{y}\right),
$$

with the imaginary field being the form $\gamma_{j}=\gamma+\delta_{j}$, where $\delta_{j}$ is uniform random real numbers within the interval $(-R, R)$, taking the role of the disorder strength. We have the mapping operator of the non-Hermitian version

$$
\begin{aligned}
\mathcal{D}_{N}(R)= & \frac{1}{2} \sum_{j=1}^{N} \prod_{l<j}\left(-\tau_{l}^{z}\right)\left[\left(h_{j}^{+}+h_{j}^{-}\right) \tau_{j}^{+}\right. \\
& \left.+\left(h_{j}^{+}-h_{j}^{-}\right) \tau_{j}^{-}\right],
\end{aligned}
$$



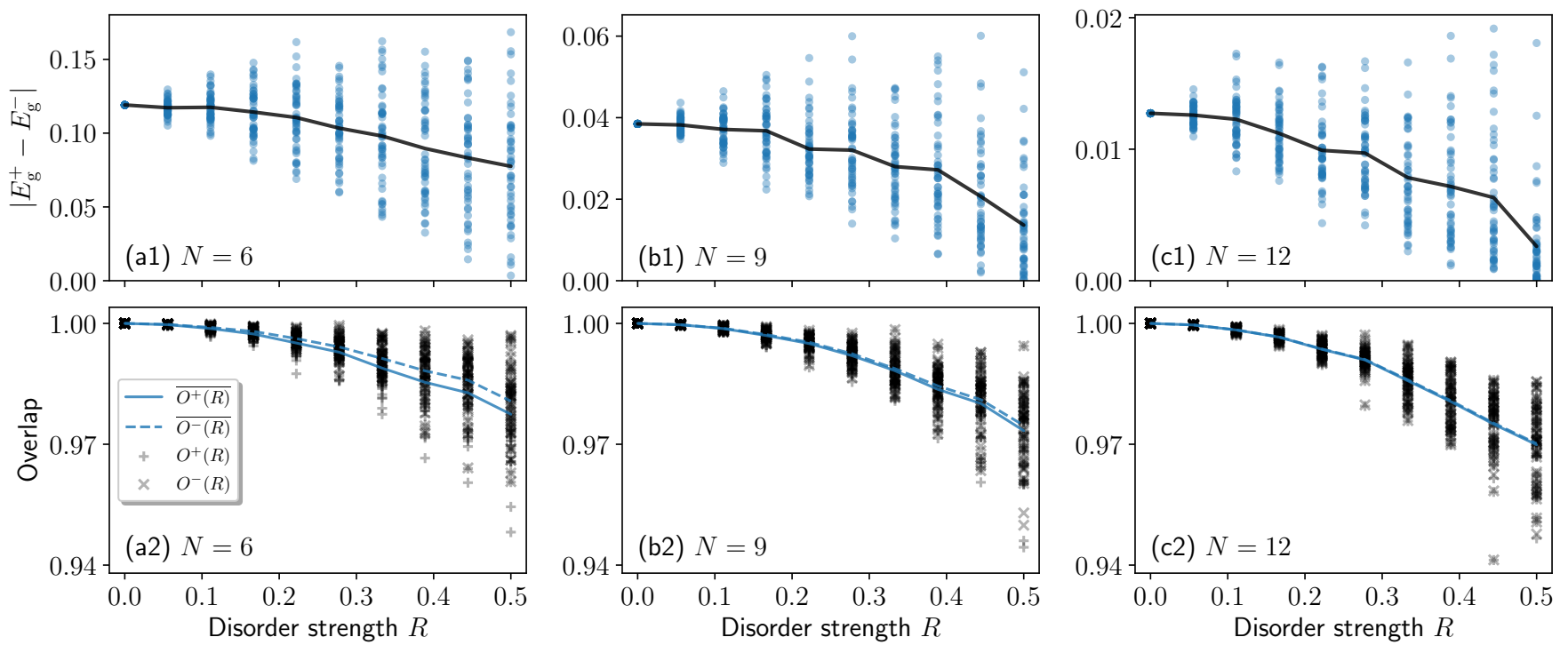

FIG. 2. Numerical results for the Hamiltonian in Eq. (27) on finite $N$ chains obtained by exact diagonalization. Panels (a1)-(c1) and (a2)-(c2) are energy gaps and the overlap $O^{ \pm}(R)$ defined in Eq. (33), respectively. For each disorder strength, numerical simulations are performed 50 times, whose average values are represented by the lines. Other parameters are $g=0.8$ and $\gamma=0.5$. The random strength $R$ is taken under the condition of a full real spectrum. Plots in panels (a1)-(c1) show that the energy gap of states $\left|\psi_{\mathrm{g}}^{+}(R)\right\rangle$ and $\left|\psi_{\mathrm{g}}^{-}(R)\right\rangle$ decreases as the size $N$ increases, which accords with the prediction for large $N$. The average values show that the energy gaps tend to become smaller as the random strength increases up to $R=0.5$. Plots in panels (a2)-(c2) show that the overlap $O^{ \pm}(R)$ decreases as the random strength $R$ increases. We find that the degeneracy is robust against large disorder, and the ground state has no evident deviation from that with zero $R$, corresponding to the uniform chain.

and its canonical conjugation

$$
\begin{aligned}
\overline{\mathcal{D}}_{N}(R)= & \frac{1}{2} \sum_{j=1}^{N} \prod_{l<j}\left(-\tau_{l}^{z}\right)\left[\left(h_{j}^{+}+h_{j}^{-}\right) \tau_{j}^{-}\right. \\
& \left.+\left(h_{j}^{+}-h_{j}^{-}\right) \tau_{j}^{+}\right],
\end{aligned}
$$

where

$$
\begin{aligned}
& h_{j}^{+}=h_{1}^{+} \prod_{m=1}^{j-1}\left(g \sqrt{1-\gamma_{m}^{2}}\right), \\
& h_{j}^{-}=h_{N}^{-} \prod_{m=j+1}^{N}\left(g \sqrt{1-\gamma_{m}^{2}}\right),
\end{aligned}
$$

and $h_{1}^{+}\left(h_{N}^{-}\right)$is determined by the normalization condition $\sum_{j=1}^{N}\left|h_{j}^{ \pm}\right|^{2}=1$. These analytical results can be obtained by the zero modes of the random core matrix

$$
\begin{aligned}
M_{\text {Ran }}= & -\frac{1}{2} \sum_{j=1}^{N-1}|2 j\rangle\langle 2 j+1| \\
& +\frac{1}{2} g \sum_{j=1}^{N} \sqrt{1-\gamma_{j}^{2}}|2 j-1\rangle\langle 2 j|+\text { H.c. }
\end{aligned}
$$

of the $2 N$-site $\mathrm{SSH}$ chain in single-particle invariant subspace or by the iterative method ${ }^{39}$. Here $\mathcal{D}_{N}(R)$ and $\overline{\mathcal{D}}_{N}(R)$ are extensions of the operators in Eqs. (22) and
(23), i.e., $\mathcal{D}_{N}=\mathcal{D}_{N}(0)$ and $\overline{\mathcal{D}}_{N}=\overline{\mathcal{D}}_{N}(0)$. The existence of ground-state degeneracy [or the relations in Eq. (26)] in the presence of nonzero $R$ depends on the existence of zero modes of the random matrix $M_{\text {Ran }}$. More specifically, if the zero modes exists, we can construct the mapping operators $\mathcal{D}_{N}(R)$ and $\overline{\mathcal{D}}_{N}(R)$ satisfying

$$
\left[\mathcal{D}_{N}(R), \mathcal{H}_{\text {Ran }}\right]=\left[\overline{\mathcal{D}}_{N}(R), \mathcal{H}_{\text {Ran }}\right]=0
$$

which is responsible for ground-state degeneracy. Here the existence of the mapping operators that satisfy the above commutation relations is also know as the normalizability of the operators ${ }^{39,40}$. It has been shown that a slight disordered perturbation prevents the left of the zero modes: in a SSH chain with non-uniform hoppings, if the average intercell hopping is stronger than the average intracell hopping, the zero modes exist in the large $N$ limit $^{10}$.

Importantly, the commutation relations in Eq. (32) can be regarded as the symmetries of the Hamiltonian $\mathcal{H}_{\text {Ran }}$. Three implications are worthy of discussion. (i) It is a conditional symmetry, which requires the parameter $|g| \sqrt{1-\gamma_{j}^{2}}<1$. (ii) It only holds in the thermodynamic limit. The two items accord with the mechanism of symmetry breaking for traditional QPT. (iii) It is a nonlocal symmetry due to the string operator $\prod_{l<j}\left(-\tau_{l}^{z}\right)$, and is robust against the local disorder. It results in topological degeneracy as topological order. In summary, the symmetries represented by commutation relations Eq. (32) 
relate two categories of QPTs in the non-Hermitian or Hermitian quantum spin system, rooted from topological order and symmetry breaking, respectively.

To demonstrate and verify the above analysis, finding out the extent of the random strength to which the degeneracy can be lifted, we perform numerical simulations for the original Hamiltonian in Eq. (27) on finite-size chain. We focus on the deviations of the ground states arising from nonzero $R$. We compute the overlap between states $\left|\psi_{\mathrm{g}}^{ \pm}\right\rangle$with zero and nonzero $R$, which is defined as

$$
O^{ \pm}(R)=\frac{\left|\left\langle\psi_{\mathrm{g}}^{ \pm}(R) \mid \psi_{\mathrm{g}}^{ \pm}\right\rangle\right|}{\left.|| \psi_{\mathrm{g}}^{ \pm}(R)\right\rangle||\left|\psi_{\mathrm{g}}^{ \pm}\right\rangle \mid}
$$

In Fig. 2, we plot the energy gap and $O^{ \pm}(R)$ for a range of $R$, which ensures all the spectra to be real. The simulations are performed by exact diagonalization of the matrices with random elements. The results are influenced by the accident from the random number generator. The average results from several random sequences show the following: (i) The energy gap decreases as the size of the chain increases, which accords with the prediction for large $N$. In the presence of the disordered field, the quasidegeneracy is not lifted, but tends to become smaller as the random strength increases up to $R=0.5$ (to ensure all $\gamma_{j}<1$ ), within the real-spectrum region. (ii) The overlap $O^{ \pm}(R)$ decreases as the random strength $R$ increases. These indicate that degeneracy is robust against large disorder, and the noise in the imaginary field does not affect the ground states too much.

\section{DISCUSSION}

In this paper, we have studied the consequence of an imaginary transverse field on the topological feature of a one-dimensional quantum Ising model. The competition between the Ising interaction and the real transverse field results in two different quantum phases with a full real spectrum, ordered and disordered. We have shown that when an imaginary field is added, the original disordered phase can be shifted to an ordered one. Although the non-Hermitian Ising model cannot support directly a Majorana fermion description, there still exists a symmetry-breaking mechanism under open boundary conditions and the thermodynamic limit. It supports the topological degeneracy due to its robustness in the presence of a random imaginary field. Our work, including the numerical result for a small-size system, reveals that disordered dissipation is constructive in establishing topological ground states, which potentially can be utilized for developing inherently robust artificial devices for topological quantum computation.

\section{ACKNOWLEDGMENTS}

This work was supported by the National Natural Science Foundation of China (under Grant No. 11874225).
* songtc@nankai.edu.cn

1 Subir S., "Quantum phase transitions," Physics World 12, 33-38 (1999).

2 X. G. Wen, "Topological orders in rigid states," Int. J. Mod. Phys. B 04, 239-271 (1990), https://doi.org/10.1142/S0217979290000139.

3 X.-G. Wen, Quantum Field Theory of Many-Body Systems: From the Origin of Sound to an Origin of Light and Electrons (Oxford University Press, Oxford, 2004).

${ }^{4}$ G. Zhang and Z. Song, "Topological characterization of extended quantum ising models," Phys. Rev. Lett. 115, 177204 (2015).

5 G. Zhang, C. Li, and Z. Song, "Majorana charges, winding numbers and chern numbers in quantum ising models," Sci. Rep. 7, 1-13 (2017).

6 A Y. Kitaev, "Unpaired majorana fermions in quantum wires," Phys. Usp. 44, 131 (2001).

7 P. Pfeuty, "The one-dimensional ising model with a transverse field," Ann. Phys. 57, 79-90 (1970).

8 W. P. Su, J. R. Schrieffer, and A. J. Heeger, "Solitons in polyacetylene," Phys. Rev. Lett. 42, 1698 (1979).

9 J. Zak, "Berry's phase for energy bands in solids," Phys. Rev. Lett. 62, 2747 (1989).

10 János K Asbóth, László Oroszlány, and András Pályi, $A$ short course on topological insulators (Springer, 2016).
11 C. Nayak, S. H Simon, A. Stern, M. Freedman, and S. D. Sarma, "Non-abelian anyons and topological quantum computation," Rev. Mod. Phys. 80, 1083 (2008).

12 A. Stern, "Non-abelian states of matter," Nature 464, 187193 (2010).

13 J. Alicea, "New directions in the pursuit of majorana fermions in solid state systems," Rep. Prog. Phys. 75, 076501 (2012).

14 J. Dalibard, Y. Castin, and K. Mølmer, "Wave-function approach to dissipative processes in quantum optics," Phys. Rev. Lett. 68, 580 (1992).

15 R. Dum, P. Zoller, and H. Ritsch, "Monte carlo simulation of the atomic master equation for spontaneous emission," Phys. Rev. A 45, 4879 (1992).

16 K. Mølmer, Y. Castin, and J. Dalibard, "Monte carlo wave-function method in quantum optics," J. Opt. Soc. Am. B 10, 524-538 (1993).

17 H. M. Wiseman, "Quantum trajectories and quantum measurement theory," Quantum Semiclass. Opt. 8, 205 (1996).

18 M. B Plenio and P. L Knight, "The quantum-jump approach to dissipative dynamics in quantum optics," Rev. Mod. Phys. 70, 101 (1998).

19 T. E Lee and C.-K. Chan, "Heralded magnetism in nonhermitian atomic systems," Phys. Rev. X 4, 041001 (2014).

20 A. Mostafazadeh, "Pseudo-hermiticity versus pt symmetry: the necessary condition for the reality of the spec- 
trum of a non-hermitian hamiltonian," J. Math. Phys. 43, 205-214 (2002).

${ }^{21}$ C. M Bender, D. C Brody, and H. F Jones, "Complex extension of quantum mechanics," Phys. Rev. Lett. 89, 270401 (2002).

22 C. M Bender, S. Boettcher, and P. N Meisinger, "Ptsymmetric quantum mechanics," J. Math. Phys. 40, 22012229 (1999).

23 C. M Bender and S. Boettcher, "Real spectra in nonhermitian hamiltonians having pt symmetry," Phys. Rev. Lett. 80, 5243 (1998).

${ }^{24}$ K. L. Zhang, H. C. Wu, L. Jin, and Z. Song, "Topological phase transition independent of system non-hermiticity," Phys. Rev. B 100, 045141 (2019).

25 P. Dorey, C. Dunning, and R. Tateo, "Spectral equivalences, bethe ansatz equations, and reality properties in pt-symmetric quantum mechanics," J Phys. A: Math Gen. 34, 5679 (2001).

26 A. Mostafazadeh, "Pseudo-hermiticity versus pt-symmetry iii: Equivalence of pseudo-hermiticity and the presence of antilinear symmetries," J. Math. Phys. 43, 3944-3951 (2002).

27 A. Mostafazadeh and A. Batal, "Physical aspects of pseudo-hermitian and pt-symmetric quantum mechanics," J. Phys. A: Math. Gen. 37, 11645 (2004).

28 A. Mostafazadeh, "Exact pt-symmetry is equivalent to hermiticity," J. Phys. A: Math Gen. 36, 7081 (2003).

29 H. F. Jones, "On pseudo-hermitian hamiltonians and their hermitian counterparts," J. Phys. A: Math Gen. 38, 1741 (2005).

30 A. Mostafazadeh, "Pseudo-hermiticity versus pt-symmetry ii. a complete characterization of non-hermitian hamiltonians with a real spectrum," J. Math. Phys. 43, 2814-2816 (2002).

31 B.-B. Wei, S.-W. Chen, H.-C. Po, and R.-B. Liu, "Phase transitions in the complex plane of physical parameters,"
Sci. Rep. 4, 5202 (2014).

32 X. Peng, H. Zhou, B.-B. Wei, J. Cui, J. Du, and R.-B. Liu, "Experimental observation of lee-yang zeros," Phys. Rev. Lett. 114, 010601 (2015).

33 B.-B. Wei, Z.-F. Jiang, and R.-B. Liu, "Thermodynamic holography," Sci. Rep. 5, 15077 (2015).

34 N. Ananikian and R. Kenna, "Imaginary magnetic fields in the real world," Physics 8, 2 (2015).

35 A. García-Saez and T.-C. Wei, "Density of yang-lee zeros in the thermodynamic limit from tensor network methods," Phys. Rev. B 92, 125132 (2015).

36 Q.-M. Chen, R.-B. Wu, T.-M. Zhang, and H. Rabitz, "Near-time-optimal control for quantum systems," Phys. Rev. A 92, 063415 (2015).

${ }^{37}$ M Krasnytska, B. Berche, Y. Holovatch, and R. Kenna, "Violation of lee-yang circle theorem for ising phase transitions on complex networks," EPL (Europhysics Letters) 111, 60009 (2015).

${ }^{38}$ P. Jordan and E. P. Wigner, "über das paulische äquivalenzverbot," in The Collected Works of Eugene Paul Wigner (Springer, 1993) pp. 109-129.

39 P. Fendley, "Strong zero modes and eigenstate phase transitions in the xyz/interacting majorana chain," J. Phys. A: Math. Theo. 49, 30LT01 (2016).

40 J. Kemp, N. Y Yao, C. R Laumann, and P. Fendley, "Long coherence times for edge spins," J. Stat. Mech. 2017, 063105 (2017).

41 D. V Else, P. Fendley, J. Kemp, and C. Nayak, "Prethermal strong zero modes and topological qubits," Phys. Rev. X 7, 041062 (2017).

42 Actually, one can develop a similar formation of Majorana fermion for the non-Hermitian spin operators $\left\{\tau_{j}^{\alpha}\right\}$. Here the Majorana fermion is no longer self-conjugate particle ${ }^{43}$.

${ }^{43}$ C. Li, X. Z. Zhang, G. Zhang, and Z. Song, "Topological phases in a kitaev chain with imbalanced pairing," Phys. Rev. B 97, 115436 (2018). 